\title{
Design instrucional orientado a artefatos: uma abordagem participativa e distribuída
}

\author{
Filipe Adeodato Garrido, Beatriz Brito do Rêgo, Ecivaldo de Souza Matos \\ ${ }^{1}$ Departamento de Ciência da Computação (DCC) - Instituto de Matemática e \\ Estatística (IME) - Universidade Federal da Bahia (UFBA) \\ Salvador - BA - Brasil \\ \{filipe.garrido, beatrizbr, ecivaldo\}@ufba.br
}

\begin{abstract}
One of the great challenges for design of online courses is the process of making instructional/educational content. Diffused (human-computer and instructional interaction) speeches in online courses may compromise the quality of the course, because it may interfere in didactic proposal or student interaction. The harmonization of these discourses is a complex activity and consequently a research challenge. Instructional design conceived from a participatory perspective among the members of the content team may favor the harmonization of discourses during the creation of the course. In this sense, this article presents a conceptual framework of online and distributed instructional design, intermediated by artifacts for designing MOOC courses. This process was evaluated during the design of a course with stakeholders geographically distributed.
\end{abstract}

Resumo. Um dos grandes desafios na concepção de cursos online é o processo de criação do conteúdo instrucional/educacional. A existência de discursos (de interação humano-computador e instrucional) difusos em cursos online pode comprometer a qualidade do curso, ao interferir no percurso instrucional ou na interação dos estudantes. A harmonização desses discursos é uma atividade complexa e, por consequência, um desafio de pesquisa. O design instrucional concebido sob uma perspectiva participativa entre os membros da equipe responsável pelo conteúdo pode favorecer a harmonização dos discursos durante a criação do curso. Nesse sentido, este artigo apresenta um framework conceitual de design instrucional distribuído e online, intermediado por artefatos para concepção de cursos MOOC. Esse processo foi avaliado durante a concepção de um curso com stakeholders geograficamente distribuídos.

\section{Introdução}

MOOC uma é modalidade de educação online, ofertada gratuitamente e sem perfil prédefinido de estudantes [Grainger 2013]. Essa modalidade tem atraído uma população diversificada com uma variedade de faixas etárias, formação, nacionalidades e objetivos educacionais.

A complexidade de harmonizar os discursos dos diversos sujeitos/papéis envolvidos na criação de um curso online constituem, há algum tempo, um importante desafio para o designer instrucional em cursos online [Filatro 2008]. 
VII Congresso Brasileiro de Informática na Educação (CBIE 2018)

Anais do XXIX Simpósio Brasileiro de Informática na Educação (SBIE 2018)

Dentre as inúmeras abordagens de design, o Design Participativo (DP) inclui os stakeholders $^{1}$ como sujeitos ativos, em vez de sujeitos consultivos, dentro do processo de design [Melo e Baranauskas 2006]. Nesse tipo de abordagem, a participação dos stakeholders costuma ocorrer desde o planejamento até a avaliação, passando por todas as fases do ciclo de design. Isso possibilita mitigar ruídos na criação de conteúdo instrucional em equipes multidisciplinares, por exemplo [Baranauskas et al. 2013].

Processos de DP podem ser instanciados com os sujeitos co-localizados ou de modo distribuído. O DP distribuído considera os stakeholders distribuídos em locais geograficamente distintos [Alves e Matos 2017b, Alves e Matos 2017a]. Todavia, segundo Alves e Matos (2017), há poucos relatos na literatura sobre o uso de DP distribuído.

No campo do Design Instrucional, acredita-se que a utilização de ferramentas computacionais em nuvem, como o Google G Suite ${ }^{2}$ (Drive, Documentos, Planilhas) e Skype $\mathrm{Web}^{3}$, em conjunto com artefatos preconcebidos para nortear o design instrucional de cursos MOOC (Massive Open Online Course) podem favorecer a criação de cursos online com a equipe distribuída em diferentes locais.

Nesse sentido, este artigo apresenta um framework conceitual de design instrucional distribuído e online, intermediado por artefatos para concepção de cursos MOOC. Esse framework foi utilizado e avaliado na concepção de um curso MOOC.

Este artigo está organizado em cinco seções. A Seção 2 apresenta aspectos conceituais do Design Participativo, Design Instrucional e artefatos para Design Instrucional. Posteriormente, a Seção 3 apresenta a metodologia de pesquisa e na Seção 4 o framework conceitual concebido durante o percurso metodológico. Por fim, a Seção 5 com as considerações finais, trabalhos futuros e agradecimentos.

\section{Fundamentação teórica}

O framework conceitual abordado neste artigo possui por fundamento o Design Participativo e o Design Instrucional, em particular o Design Instrucional Contextualizado. Diante disto, as subseções seguintes explanam sobre ambos.

\subsection{Design Participativo}

O Design Participativo (DP) é uma abordagem clássica de design, cujo principal objetivo/diferencial é a inclusão dos sujeitos-usuário na concepção dos artefatos [Spinuzzi 2005, Melo e Baranauskas 2006].

O DP é sustentado pelo princípio do envolvimento de diferentes usuários durante as diferentes etapas de projeto, considerando as potenciais contribuições compartilhadas para o resultado do projeto [Luck 2003]. Logo, o resultado final de um projeto concebido à luz do DP será influenciado pela diversidade de sujeitos envolvidos nas tomadas de decisão de design.

Entretanto, um processo de DP não é caracterizado simplesmente por colocar os usuários em contato com os designers, mas em proporcionar um espaço para que existam opiniões e críticas de ambos (usuários e designers) sem hierarquização ou distinção

\footnotetext{
${ }^{1}$ Partes interessadas e impactadas. (grifo nosso)

${ }^{2}$ https://gsuite.google.com.br/intl/pt-BR/

${ }^{3}$ https://web.skype.com/
} 
VII Congresso Brasileiro de Informática na Educação (CBIE 2018)

Anais do XXIX Simpósio Brasileiro de Informática na Educação (SBIE 2018)

da atuação [Luck 2003]; sem deixar de considerar que usuários não são designers, nem designers são usuários [Melo e Baranauskas 2006].

As técnicas para DP promovem atuação direta do usuário em várias etapas do processo de design, como na identificação e clarificação do problema, requisitos \& análise, design de alto nível, design detalhado, avaliação, customização pelo usuário e (re)design [Melo e Baranauskas 2006]. Isso permite aos usuários assumirem o papel de "co-designer"[Águas 2012]. Não existe uma hierarquia no DP, todos atuam em condições de igualdade em prol do alcance dos objetivos de design [Baranauskas et al. 2013].

\subsection{Design Instrucional}

Definido pela Association for Educational Communications and Technology (AECT) como a teoria e prática de design, desenvolvimento, utilização, gerenciamento, e avaliação de processos e recursos para a aprendizagem [Reiser 2001, p. 1]; o Design Instrucional (DInst) é a ciência da criação de currículo de instrução que se destina a produzir resultados de aprendizagem específicos, baseados não apenas na pesquisa pedagógica, mas também nas práticas de instrução contemporâneas [Filatro e Piconez 2004].

Entretanto, o DInst não possui relação direta com o desenvolvimento de software educacional ou design gráfico, essas habilidades são úteis à produção de um currículo efetivo, razão pela qual, geralmente, indivíduos com essas habilidades são incluídos como membros das equipes. Cabe ao designer instrucional gerenciar os diferentes atores, com seus respectivos papéis (responsabilidades e atribuições), harmonizando os discursos e guiando a produção da proposta didática [Filatro e Piconez 2004, p. 135-141].

No projeto de cursos online, o DInst se ocupa do design de artefatos digitais com uso das Tecnologias de Informação e Comunicação (TIC) para representar elementos e processos a ser realizados em ambientes de aprendizagem. Segundo Filatro, há diferentes modelos de design, a depender do contexto de utilização, dos quais se destacam os modelos: fixo, aberto e contextualizado [Filatro 2008, p. 26].

Um dos modelos mais utilizados é o ADDIE (Analysis, Design, Development, Implementation e Evaluation) [Branson et al. 1975]. Destacam-se ainda outros modelos empregados com frequência em DInst, como o modelo baseado na Taxonomia de Bloom ou o ARCS [Filatro e Piconez 2004, Ferraz et al. 2010].

O Design Instrucional Contextualizado (DIC), uma proposta de DInst com as mesmas fases do modelo ADDIE, possui como diferencial a centralização do processo em avaliações realizadas ao longo de todo o design instrucional, influenciando modificações no conteúdo e considerando a importância da interação entre designers-estudantes e o feedback formativo [Filatro e Piconez 2004].

No DIC as situações didáticas ocorridas ao longo do percurso determinam as diretrizes de design. A entrega de unidades instrucionais são menores em comparação aos outros dois modelos, aberto e fixo. Para tanto, o DIC utiliza o contexto como elemento basilar para modificar a composição do conteúdo, avaliações e interações. Logo, nesse modelo, a interação entre os estudantes teria a capacidade de modificar o conteúdo disponibilizado no curso [Filatro e Piconez 2004, Filatro 2008]. 
VII Congresso Brasileiro de Informática na Educação (CBIE 2018)

Anais do XXIX Simpósio Brasileiro de Informática na Educação (SBIE 2018)

\section{Metodologia}

O Design Instrucional não exige ou indica o uso de algum método, técnica ou ferramenta para o projeto de design. Ele apresenta elementos para articulação entre a forma (processo) e a função (artefatos), a fim de que se cumpram os objetivos educacionais propostos [Filatro e Piconez 2004, p. 56].

$\mathrm{Na}$ abordagem apresentada neste artigo, os artefatos são utilizados como ferramentas/recursos epistêmicos para nortear a compreensão da equipe compositora do conteúdo, a saber: designer instrucional, professora (conteudista) e administrador da plataforma de MOOC. Para tanto, em momentos distintos, apoiamos o processo de design nos seguintes artefatos, por ordem de sequência de uso:

- MOOC Canvas (cf. Subseção 3.1);

- Matriz Instrucional ( $c f$. Subseção 3.2);

- Checklist para avaliação de Design Instrucional (cf. Subseção 3.4).

\subsection{Analisar}

Como preconizado pelo DIC, analisar a situação didática proposta, compreendendo os agentes pedagógicos envolvidos, as ferramentas, o ambiente, recursos disponíveis, e demais aspectos relevantes para o processo de design compõem a primeira fase, denominada análise [Filatro 2008].

Nessa fase, realizou-se um diálogo com o conteudista via Skype ${ }^{4}$, sob a perspectiva da técnica contextual inquiry (investigação contextual). Sendo o objetivo desse primeiro encontro a identificação do perfil do conteudista, expertise e áreas de atuação, suas propostas para concepção do curso e como seriam as etapas seguintes, intermediadas por artefatos computacionais.

A contextual inquiry é uma técnica de natureza etnográfica, com a qual os designers levantam dados por meio da observação dos usuários enquanto eles executam determinadas atividades do seu contexto [Muller et al. 1997]. Com o resultado da observação é possível que os designers identifiquem as necessidades do usuário de modo contextualizado e mais próximo da realidade [Rosa e Matos 2016].

Durante esse diálogo, as partes, designer e conteudista, uniram-se como codesigners, realizando as atividades inerentes à concepção do curso sem uma hierarquia definida [Baranauskas et al. 2013]. Ao final dessa etapa foi apresentado o artefato intitulado MOOC Canvas ( $c f$. Figura 1). Sendo um artefato baseado numa ferramenta de gerenciamento estratégico, que permite desenvolver e esboçar modelos novos ou existentes de negócio [Osterwalder e Pigneur 2010]. O MOOC Canvas é uma estrutura simples e visual para educadores que precisam criar um MOOC [Alario Hoyos et al. 2014].

Como pode ser visto na Figura 1, há onze campos que devem ser preenchidos pela equipe de designers. Esses campos se classificam em duas categorias, recursos disponíveis e decisões de designer. A primeira categoria refere-se aos recursos-chave que o corpo docente tem à sua disposição no momento de projeto do MOOC, referentes aos quadrados sombreados em cinza. As decisões de design encontram-se no espaço com fundo branco. Referem-se às respostas que irão impactar no design do curso [Alario Hoyos et al. 2014].

\footnotetext{
${ }^{4}$ https://web.skype.com.br/
} 
VII Congresso Brasileiro de Informática na Educação (CBIE 2018)

Anais do XXIX Simpósio Brasileiro de Informática na Educação (SBIE 2018)

Além da tradução e adaptação dos termos utilizados no MOOC Canvas, alguns tópicos tiveram seus elementos de preenchimento modificados por existir o apoio de outro artefato, a matriz de design instrucional. Verificado nos campo 9 e 10 - Conteúdos de aprendizagem e Atividades de avaliação, respectivamente, que foram mais detalhados na matriz. Apesar de manter o campo 6 - Perfil dos estudantes (público-alvo), não utilizou-se por acreditarmos que MOOC não possuem tal restrição, como indicado por [Grainger 2013].

Após essa fase, pode-se iniciar o processo de desenho, gerando um rascunho ou primeira versão do curso. Os detalhes desse processo são apresentados na subseção seguinte.

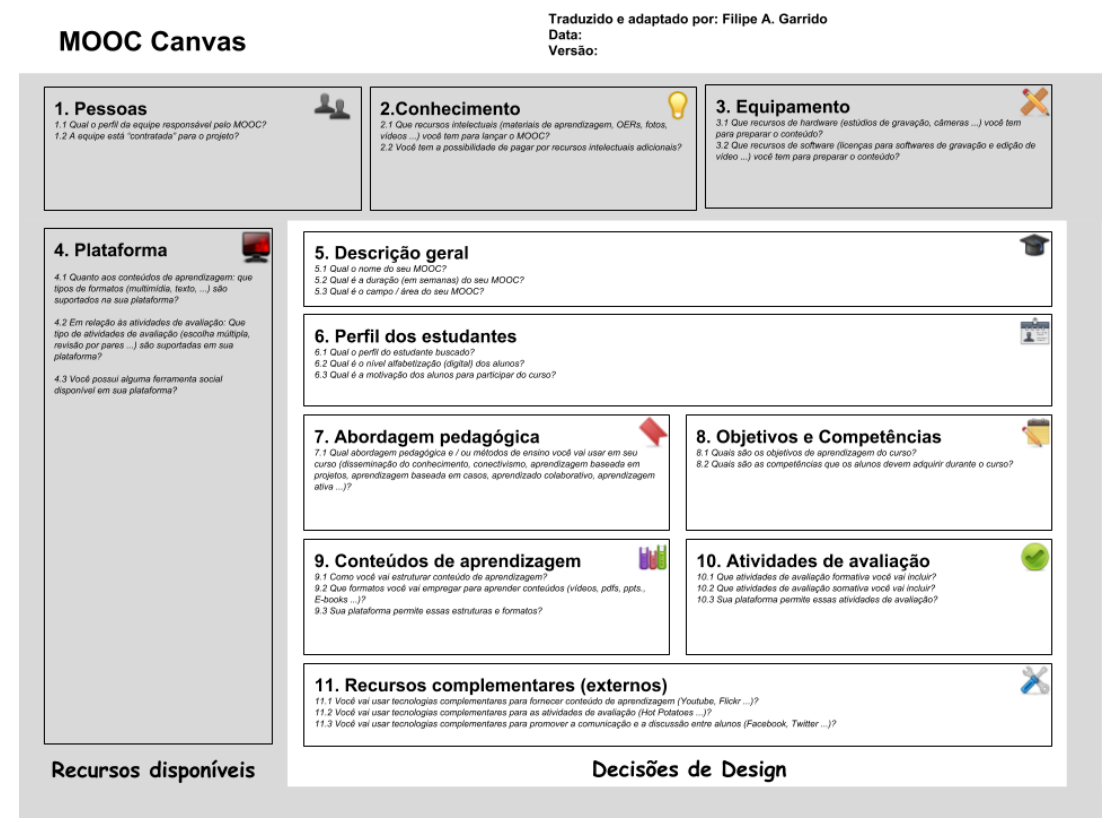

Figura 1. MOOC Canvas traduzido e adaptado de [Alario Hoyos et al. 2014]

\subsection{Desenhar}

Nesta fase, a revisão do MOOC Canvas preenchido por completo suportou as decisões da equipe. O preenchimento ocorreu de forma assíncrona, entre o designer instrucional e o conteudista. Entretanto, o esboço (desenho) do curso, conteúdo e suas divisões, avaliações, recursos e interações, foi discutido por ambos durante a revisão do MOOC Canvas por completo.

O preenchimento foi executado com a utilização de ferramentas colaborativas online. O pacote Google G Suite ${ }^{5}$ foi selecionado devido à familiaridade do conteudista com seu uso e seu grande número de utilizadores, facilitando a replicação do processo. Para o MOOC Canvas utilizou-se o Google Drive e a ferramenta Desenhos.

Posteriormente, o conteúdo completo do curso foi criado pelo conteudista utilizando o Google Documentos. Permitindo a visualização e contribuição, por meio de comentários e edições no texto, dos demais stakeholders caso fosse necessário. Entretanto, o conteúdo carecia de uma divisão fluída para favorecer a aprendizagem do estudante.

\footnotetext{
${ }^{5} \mathrm{https}: / /$ gsuite.google.com/intl/pt-BR/features/
} 
VII Congresso Brasileiro de Informática na Educação (CBIE 2018)

Anais do XXIX Simpósio Brasileiro de Informática na Educação (SBIE 2018)

A matriz instrucional (ver Figura 2), segundo artefato dessa abordagem, foi apresentado ao conteudista para amparar a divisão do curso em unidades instrucionais menores, organizando o pretenso percurso didático [Filatro 2008, p. 44].

Sua estrutura é composta por unidades instrucionais, objetivos, papéis, atividades, duração, ferramentas, conteúdos e avaliação. A matriz possuía notas explicativas para sanar possíveis dúvidas sobre o preenchimento. Para a matriz de design instrucional utilizou-se a ferramenta Google Planilhas.

As unidades instrucionais (módulos) e suas atividades, ferramentas e avaliações indicam o tipo de ambiente virtual de aprendizagem que será adequado para o curso. Por consequência, repercutem na fase de desenvolvimento.

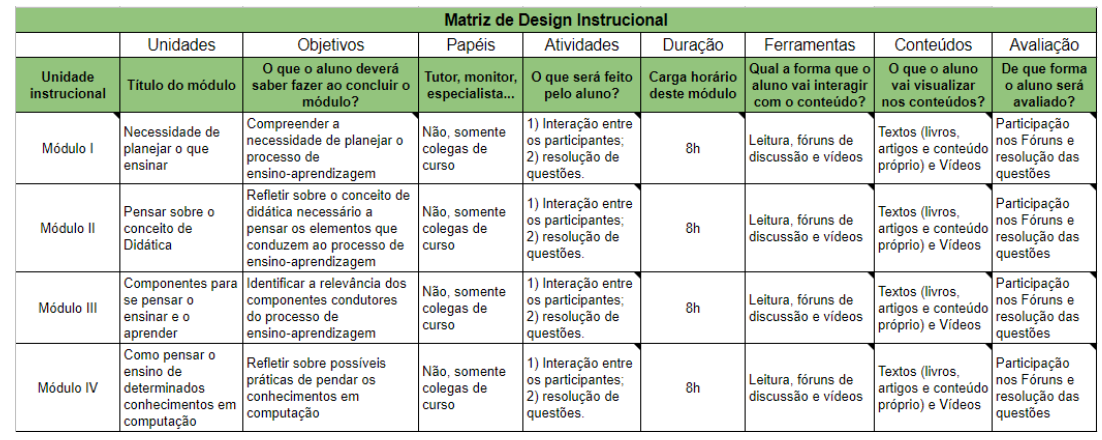

Figura 2. Matriz instrucional preenchida pelo conteudista

\subsection{Desenvolver \& Implementar}

A estruturação do curso no ambiente MOOC que o hospedou foi desenvolvida com base no MOOC Canvas e na matriz de design instrucional. Portanto, os artefatos serviram como ferramentas epistêmicas para conceber o curso.

O desenvolvimento foi feito diretamente no ambiente Moodle, unificando essa etapa com a implementação. Uma vez que ambos ocorreram em momento único, sem intervalo entre etapas.

O Moodle (Modular Object-Oriented Dynamic Learning Environment), seguindo as diretrizes de um MOOC, foi selecionado para uso neste estudo de acordo com os mesmos critérios utilizados para escolha do Google G Suite: quantidade de usuários e familiaridade dos designers. Outros ambientes educacionais online, como Udemy ${ }^{6}$ ou Google Classroom $^{7}$ também foram considerados aptos para a proposta.

O conteudista percebeu a necessidade da inclusão de mais material multimídia para o conteúdo, expandindo com vídeos disponibilizados no Youtube ${ }^{8}$. Esses vídeos são considerados fonte externa ao conteúdo, assim como textos disponíveis integralmente no curso e que não são de autoria do conteudista.

Cada elemento do curso que integra um módulo, com seus respectivos objetivos, atividades e avaliações, foi validado. A matriz de design instrucional serviu como guia de

\footnotetext{
${ }^{6}$ https://www.udemy.com/

${ }^{7}$ https://classroom.google.com/

${ }^{8} \mathrm{https}: / /$ www.youtube.com/
} 
VII Congresso Brasileiro de Informática na Educação (CBIE 2018)

Anais do XXIX Simpósio Brasileiro de Informática na Educação (SBIE 2018)

referência para verificar se os elementos implementados estavam em conformidade com o desenho, etapa anterior.

Instrumentos de avaliação para o curso, como testes de múltipla escolha e fórum avaliativo, foram definidos entre os módulos. Nesse processo, a auto-avaliação é elemento-chave, estimulando a avaliação em pares. Os resultados desses testes serão analisados após a conclusão do curso, ainda em andamento, para fins de validação do design instrucional por parte dos estudantes.

O curso projetado foi dividido em quatro módulos: (i) Necessidades de planejar o que ensinar; (ii) Pensar sobre conceito de didática, (iii) Componentes para se pensar o ensinar e o aprender; e (iv) Como pensar o ensino de determinados conhecimentos em computação.

Contudo, o curso criado no Moodle, até esse momento, possui caráter experimental. Uma vez que ainda não concluiu a implementação, na qual ocorre a ambientação à proposta de design instrucional. Nesse estudo, a realização da validação ocorre na fase de avaliação, por intermédio de simulação da situação de ensino-aprendizagem com o conteudista.

\subsection{Avaliar}

Essa fase do framework conceitual tratou da avaliação pelo conteudista da proposta de design instrucional. Uma vez que as avaliações do curso, sob a perspectiva do estudante, foram retratadas na subseção anterior.

O protocolo verbal think-aloud é um método que provoca a fala nos seus participantes, fazendo com que eles verbalizem o que estão pensando, sentindo e fazendo ao usar o sistema determinado na avaliação. Os participantes são geralmente orientados a "pensar em voz alta", agindo como se estivessem sozinhos em um ambiente, falando para si mesmos [Markopoulos et al. 2008]. Todo esse processo deve ser gravado para depois ser analisado pelo executor do método.

Após o desenvolvimento e implementação do curso no Moodle, ocorreu a execução do think-aloud. Os comentários do conteudista durante a interação com o curso de forma análoga ao percurso didático do estudante foram gravados para análise. Complementarmente ao think-aloud, uma inspeção foi realizada por meio de uma checklist fundamentada no framework criado por Charlotte Danielson [Danielson 2011, Danielson 2013]. Esse framework é um conjunto de componentes instrucionais fundamentados na visão construtivista de aprendizagem.

A checklist disponibilizada pela Universidade de New Hampshire sob uma licença aberta, foi traduzida e adaptada para esse estudo (http://bit.ly/2D8Mve5). Com um total de 47 tópicos (45 no original), sendo 28 considerados essências (17 no original). A atribuição de notas por tópico é entre 0 e 2 pontos, para cada item avaliado. Existe ainda a opção Não se aplica (N/A) com valor 0.

O conteudista, por meio do Google Planilhas, preencheu os campos correspondentes aos tópicos na checklist e atribuiu 91 pontos ( $97 \%$ do total) em sua avaliação. Entre os tópicos considerados essenciais o total de pontos atribuídos foi de 55 pontos (98\% do total possível). Ressaltamos que os campos referentes à acessibilidade foram opcionais neste momento. $\mathrm{O}$ ambiente em que estão inseridos os cursos possui recursos de acessibilidade 
VII Congresso Brasileiro de Informática na Educação (CBIE 2018)

Anais do XXIX Simpósio Brasileiro de Informática na Educação (SBIE 2018)

nativo.

\section{Framework conceitual}

O design orientado a artefatos apresentado neste artigo se baseia no modelo DIC [Filatro e Piconez 2004] articulado ao espaço de design [Silva 2010]. Consideramos esse modelo como um framework ${ }^{9}$ conceitual, constituído por um conjunto de conceitos usado para resolver um problema de um domínio específico.

O espaço de problema agrega os elementos anteriores ao artefato a ser desenvolvido, constituído por: contexto em que o sistema será utilizado; domínio em que pertence o usuário; e seus objetivos. O espaço de solução, por sua vez, é a porção de atuação do designer, onde se encontram os recursos envolvidos para apoiar as decisões do designer [Silva 2010]. O framework conceitual considera as fases do DIC e seus elementos são artefatos, técnicas e ferramentas computacionais.

A intersecção entre o espaço de design e o modelo DIC, perceptível na divisão entre as fases que compõem o processo, oportunizou a criação e execução desse framework ( $c f$. Figura 3). Por isso, a metodologia deste estudo foi dividida em 4 partes: analisar; desenhar; desenvolver \& implementar; e avaliar.

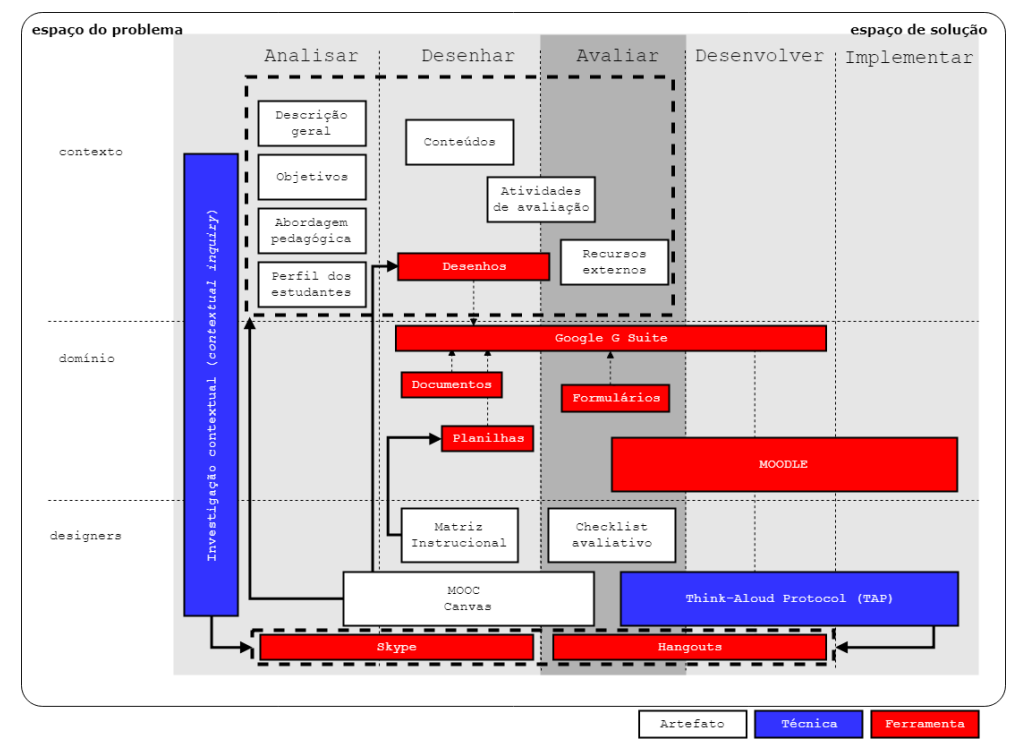

Figura 3. Framework conceitual (Espaço de design do estudo)

Destaca-se que o framework conceitual não se trata de um modelo de processo rígido, podendo adaptar-se à inclusão ou exclusão de métodos, técnicas ou ferramentas para o objetivo pretendido com seu uso.

\section{Considerações finais e trabalhos futuros}

Esse artigo apresentou um framework conceitual de design instrucional distribuído para concepção participativa de um curso MOOC. Foram apresentadas as etapas do framework conceitual, baseado no modelo de Design Instrucional Contextualizado articulado ao espaço de design.

\footnotetext{
${ }^{9}$ Framework é um conjunto de classes que colaboram para realizar uma responsabilidade para um domínio de um subsistema da aplicação [Fayad e Schmidt 1997].
} 
VII Congresso Brasileiro de Informática na Educação (CBIE 2018)

Anais do XXIX Simpósio Brasileiro de Informática na Educação (SBIE 2018)

Ao concluir todas as fases que compõem o framework conceitual proposto para esse estudo, o curso estará finalizado e pronto para ser disponibilizado aos estudantes do MOOC. Ademais, o conjunto de artefatos, técnicas e ferramentas compositores do framework conceitual consolidam um espaço de design coeso e propício para a atividades inerentes ao design instrucional.

Os artefatos traduzidos, adaptados e utilizados nesse processo serviram como ferramentas epistêmicas para a equipe de designers; reafirmando o potencial de ferramentas computacionais em nuvem no favorecimento do design participativo distribuído, independente do domínio de aplicação. A replicação do processo com outro professor, instrutor ou conteudista que não tenha concebido conteúdo para cursos online ou sem familiaridade com ferramentas computacionais, utilizadas no framework, é desejada para validar a efetividade do processo de design com diferentes perfis de profissionais. Espera-se evoluir o processo, atualizando os artefatos utilizados, quando necessário.

Para trabalhos futuros, pretende-se realizar novos ciclos de avaliação do framework, de modo que seja possível evidenciar os seus limites metodológicos e epistemológicos. Pretende-se também desenvolver uma ferramenta que suporte a concepção do design de interação sob a perspectiva do percurso instrucional, possibilitando a indicação de um caminho ideal de favorecimento da aprendizagem em tempo de interação. De modo que seja possível idealizar um design de interação que preze pela alta comunicabilidade do sistema.

Por fim, a avaliação do curso pelo conteudista por meio da checklist, foi essencial para validar a efetividade do processo utilizado e insights para futuras atualizações do curso, como indicado pelo DIC. Além disso, pretende-se avaliar o percurso instrucional dos estudantes para verificar a influência dessa proposta na permanência dos estudantes em cursos MOOC; bem como sua avaliação sobre o design instrucional do curso resultante.

\section{Agradecimentos}

Agradecemos aos membros do Grupo de Pesquisa e Extensão em Informática, Educação e Sociedade - Onda Digital e à Fundação de Amparo à Pesquisa do Estado da Bahia (FAPESB) pelo financiamento desta pesquisa.

\section{Referências}

Águas, S. (2012). Do design ao co-design: uma oportunidade de design participativo na transformação do espaço público. On the W@ terfront. Public Art. Urban Design. Civic Participation. Urban Regeneration, (22):57-70.

Alario Hoyos, C., Pérez Sanagustín, M., Cormier, D., e Delgado Kloos, C. (2014). Proposal for a conceptual framework for educators to describe and design moocs. Journal of Universal Computer Science (JUCS), 20(1):6-23.

Alves, D. D. e Matos, E. S. (2017a). Desafios no uso de design participativo em ambientes distribuídos de desenvolvimento de software educacional livre. Revista de Sistemas e Computação-RSC, 7(2).

Alves, D. D. e Matos, E. S. (2017b). Design participativo em ambientes distribuídos de desenvolvimento de software educacional livre: desafios de pesquisa. Anais dos Workshops do Congresso Brasileiro de Informática na Educação, 6(1):1354. 
VII Congresso Brasileiro de Informática na Educação (CBIE 2018)

Anais do XXIX Simpósio Brasileiro de Informática na Educação (SBIE 2018)

Baranauskas, M. C. C., Martins, M. C., e Valente, J. A. (2013). Codesign de Redes Digitais: tecnologia e educação a serviço da inclusão social. Penso Editora.

Branson, R. K., Rayner, G. T., Cox, J. L., Furman, J. P., e King, F. (1975). Interservice Procedures for Instructional Systems Development. Phase 4 and 5. Implement and Control. Technical report, Florida State Univ Tallahassee Center For Educational Technology (ED 122 022).

Danielson, C. (2011). Enhancing professional practice: A framework for teaching. Association for Supervision and Curriculum Development - ASCD.

Danielson, C. (2013). The framework for teaching evaluation instrument, 2013 instructionally focused edition. Retrieved January, 17:2017.

Fayad, M. e Schmidt, D. C. (1997). Object-oriented application frameworks. Communications of the ACM, 40(10):32-38.

Ferraz, A., Belhot, R. V., et al. (2010). Taxonomia de bloom: revisão teórica e apresentação das adequações do instrumento para definição de objetivos instrucionais. Gestão Produção, São Carlos, 17(2):421-431.

Filatro, A. (2008). Design instrucional na prática. Pearson Education do Brasil.

Filatro, A. e Piconez, S. C. B. (2004). Design instrucional contextualizado. São Paulo: Senac.

Grainger, B. (2013). Introduction to moocs: avalanche, illusion or augmentation. Policy Brief - UNESCO (july). Institute for Information Technologies in Education. URL: http://iite. unesco. org/pics/publications/en/files/3214722. pdf.

Luck, R. (2003). Dialogue in participatory design. Design studies, 24(6):523-535.

Markopoulos, P., Read, J. C., MacFarlane, S., e Hoysniemi, J. (2008). Evaluating children's interactive products: principles and practices for interaction designers. Elsevier.

Melo, A. M. e Baranauskas, M. C. C. (2006). Design para a inclusão: desafios e proposta. Anais VII Simpósio Brasileiro sobre Fatores Humanos em Sistemas Computacionais, pages 11-20.

Muller, M. J., Haslwanter, J. H., e Dayton, T. (1997). Participatory practices in the software lifecycle. In Handbook of Human-Computer Interaction (Second Edition), pages 255-297. Elsevier.

Osterwalder, A. e Pigneur, Y. (2010). Business model canvas. Self published. Last.

Reiser, R. A. (2001). A history of instructional design and technology: Part i: A history of instructional media. Educational technology research and development, 49(1):53.

Rosa, J. e Matos, E. (2016). Semio-participatory framework for interaction design of educational software. Proceedings of the 15th Brazilian Symposium on Human Factors in Computer Systems, page $\mathrm{n}^{\mathrm{o}} 33$.

Silva, B. S. (2010). O Uso de Casos na Reflexão em Ação em Atividades de Design de IHC. PhD thesis, Pontifícia Universidade Católica do Rio de Janeiro (PUC-Rio).

Spinuzzi, C. (2005). The methodology of participatory design. Technical communication, 52(2):163-174. 